\title{
Equilibrium solutions of relativistic rotating stars with mixed poloidal and toroidal magnetic fields
}

\author{
Kōji Uryū, 1 , 团 Eric Gourgoulhon, 2, 团 Charalampos M. Markakis, ${ }^{3,4}$, 团 \\ Kotaro Fujisawa,, , Antonios Tsokaros, ${ }^{6,9}$ and Yoshiharu Eriguchi, ${ }^{7}, * *$ \\ ${ }^{1}$ Department of Physics, University of the Ryukyus, \\ Senbaru 1, Nishihara, Okinawa 903-0213, Japan \\ ${ }^{2}$ Laboratoire Univers et Théories, UMR 8102 du CNRS, \\ Observatoire de Paris, Université Paris Diderot, F-92190 Meudon, France \\ ${ }^{3}$ Theoretical Physics Institute, University of Jena, Max-Wien-Platz 1, 07743 Jena, Germany \\ ${ }^{4}$ Mathematical Sciences, University of Southampton, Southampton, SO17 1BJ, United Kingdom \\ ${ }^{5}$ Advanced Research Institute for Science and Engineering, \\ Waseda University, 3-4-1 Okubo, Shinjuku-ku, Tokyo 169-8555, Japan \\ ${ }^{6}$ Institut für Theoretische Physik, Johann Wolfgang Goethe-Universität, \\ Max-von-Laue-Str. 1, 60438 Frankfurt am Main, Germany \\ ${ }^{7}$ Department of Earth Science and Astronomy, Graduate School of Arts and Sciences, \\ University of Tokyo, Komaba 3-8-1, Meguro, 153-8902 Tokyo, Japan
}

(Dated: June 15, 2018)

\begin{abstract}
Stationary and axisymmetric solutions of relativistic rotating stars with strong mixed poloidal and toroidal magnetic fields are obtained numerically. Because of the mixed components of the magnetic field, the underlying stationary and axisymmetric spacetimes are no longer circular. These configurations are computed from the full set of the Einstein-Maxwell equations, Maxwell's equations and from first integrals and integrability conditions of the magnetohydrodynamic equilibrium equations. After a brief introduction of the formulation of the problem, we present the first results for highly deformed magnetized rotating compact stars.
\end{abstract}

Introduction: Neutron stars (NS) with strong surface magnetic fields around $10^{14}-10^{15} \mathrm{G}$ are considered as the source of soft gamma repeaters and anomalous x-ray pulsars [1]. The widely accepted magnetar model stimulated an interest in constructing solutions of strongly magnetized relativistic rotating stars. Numerical computations of such magnetized stars are useful tools for investigating allowed configurations of the interior magnetic fields, or a limit of the strength of the fields. In particular, compact stars with stronger magnetic fields could model newly born magnetars (see, e.g. 2]).

An approach for studying magnetized rotating neutron stars is to obtain their equilibrium configurations. Stationary and axisymmetric solutions of rotating relativistic stars with strong poloidal fields have been successfully calculated in [3], and with toroidal fields in [4, 5]. In these computations, the spacetime is assumed to be orthogonally transitive (circular - invariant under a simultaneous inversion of $t \rightarrow-t$, and $\phi \rightarrow-\phi$ ), because the configuration of magnetic fields is restricted to either purely poloidal or toroidal fields and the velocity field is to circular [6 9] A relativistic formulation for more general magnetized relativistic stars with mixed poloidal and toroidal fields has been derived by Bekenstein and Oron

\footnotetext{
* uryu@sci.u-ryukyu.ac.jp

$\dagger$ eric.gourgoulhon@obspm.fr

$\ddagger$ charalampos.markakis@uni-jena.de

$\S$ fujisawa@heap.phys.waseda.ac.jp

ฯ tsokaros@th.physik.uni-frankfurt.de

** eriguchi@ea.c.u-tokyo.ac.jp
}

[10]. The formulation has been used to obtain slowly rotating and weakly magnetized solutions [11, 12], and has been improved for obtaining more stable solutions [13]. More recently, numerical solutions for mixed poloidal and toroidal fields have been obtained under a simplified relativistic gravity [14] 1

In this paper, we present a formulation and numerical solutions for rapidly rotating relativistic stars with strong mixed poloidal and toroidal magnetic fields. Our solutions extend the above works 11,14 to highly deformed configurations as a result of rapid rotation and stronger magnetic fields, which can not be calculated from perturbative methods. We assume stationarity and axisymmetry of the gravitational, electromagnetic and matter fields, but we do not assume the spacetime to be circular, or spatially conformally flat. Moreover our new method of solving Einstein's and Maxwell's equations are not restricted to axial symmetry. The first integrals of a system of relativistic magnetohydrodynamic (MHD) equations are derived assuming the above symmetries as well as perfect conductor conditions [10, 11, 15], and solved to obtain self-consistent, non-perturbative, equilibrium configurations for the first time.

The existence and stability of mixed poloidal and toroidal magnetic fields in compact stars is supported by a number of recent simulations of magnetized rotating stars 16. It is demonstrated that such stable configurations of mixed fields are reached from arbitrary ini-

\footnotetext{
1 They have assumed the form of spacetime metric to be $d s^{2}=$ $-\alpha^{2} d t^{2}+\psi^{4} f_{i j} d x^{i} d x^{j}$, where $f_{i j}$ is a flat spatial metric.
} 
tial data. Several groups have been developing relativistic MHD simulation codes, and have performed some, rather preliminary, simulations of magnetized compact stars [17]. Our equilibrium solutions provide initial data for such simulations aiming to obtain stable configurations of strongly magnetized compact stars. The stability of equilibrium solutions may be determined from approaches complementary to the simulations, such as an application of a linear perturbation method described in [18].

In this paper, we use $G=c=4 \pi \epsilon_{0}=1$ units unless otherwise specified. Greek indices, $\alpha, \beta, \ldots$ are used for spacetime tensors, lower case latin indices, $a, b, \ldots$ for 3 dimensional spatial tensors, and upper case Latin indices, $A, B, \ldots$ for 2-dimensional spatial tensors in a meridional plane. The exterior derivative of a 1-form $w_{\alpha}$ is denoted by $(d w)_{\alpha \beta}=\nabla_{\alpha} w_{\beta}-\nabla_{\beta} w_{\alpha}$.

Formulation: A relativistic rotating star with both poloidal and toroidal magnetic fields (and possibly with electric fields and/or meridional flows) can be modeled by Einstein-Maxwell charged magnetized perfect-fluid spacetimes. Although stationarity and axisymmetry is assumed for spacetime, electromagnetic fields and matter, such spacetimes cannot be circular2 Such noncircular spacetimes have been calculated in the slow rotation and weak magnetic field limit as in [11 13], and in the fully nonlinear regime for non-magnetized compact star with large meridional flow in 24. A proposed formulation for computing non-circular spacetimes invokes a $2+1+1$ spacetime decomposition [24, 25]. Here, instead of this formulation, we use the one developed in [26], which is based on a $3+1$ decomposition and has been successfully applied for computing waveless initial data for binary neutron stars (BNS) [27].

The spacetime $\left(\mathcal{M}, g_{\alpha \beta}\right), \mathcal{M}=\mathbb{R} \times \Sigma$, is foliated by a family of spacelike hypersurfaces $\Sigma_{t}=\chi_{t}\left(\Sigma_{0}\right)$ where the hypersurface $\Sigma=\Sigma_{0}$ is an initial slice. The diffeomorphism $\chi_{t}$ is generated by the time symmetry vector $t^{\alpha}$, so $\Sigma_{t}$ are identical for any $t$. The timelike vector $t^{\alpha}$ is tangent to the curves $t \rightarrow(t, x) \in \mathbb{R} \times \Sigma$, and is related to the future-pointing normal $n^{\alpha}$ of $\Sigma_{t}$ by $t^{\alpha}=\alpha n^{\alpha}+\beta^{\alpha}$, where $\alpha$ is the lapse and $\beta^{\alpha}$ the shift satisfying $\beta^{\alpha} n_{\alpha}=0$. The projection tensor $\gamma_{\alpha \beta}=g_{\alpha \beta}+n_{\alpha} n_{\beta}$ restricted to a slice $\Sigma_{t}$ is equal to a spatial metric $\gamma_{a b}(t)$ on $\Sigma_{t}$. We introduce a conformally rescaled spatial metric $\tilde{\gamma}_{a b}=\psi^{-4} \gamma_{a b}$ whose decomposition is specified by the condition $\operatorname{det} \tilde{\gamma}_{a b}=\operatorname{det} f_{a b}$, where $f_{a b}$ is a flat metric on $\Sigma_{t}$. Then, the metric $g_{\alpha \beta}$ in $3+1$ form becomes $d s^{2}=-\alpha^{2} d t^{2}+\psi^{4} \tilde{\gamma}_{a b}\left(d x^{a}+\beta^{a} d t\right)\left(d x^{b}+\beta^{b} d t\right)$.

2 Stationary and axisymmetric spacetime is called circular if the 2 -planes orthogonal to two killing vectors $t^{\alpha}$ and $\phi^{\alpha}$ is integrable. The derivation of the metric for circular spacetime is found in [6, 8, 9, 19 [22]. It relies on the vanishing twists of two killing vectors $t^{\alpha}$ and $\phi^{\alpha}, t_{[\alpha} \phi_{\beta} \nabla_{\gamma} t_{\delta]}=0$ and $t_{[\alpha} \phi_{\beta} \nabla_{\gamma} \phi_{\delta]}=0$ (Frobenius conditions), which are no longer satisfied for the spacetime associated with mixed poloidal and toroidal fields [7, 23].
The metric potentials $\left\{\psi, \beta_{a}, \alpha, \tilde{\gamma}_{a b}\right\}$ are solved from the respective components of the $3+1$ Einstein-Maxwell equations, which consist of the Hamiltonian and momentum constraints, the spatial trace part of EinsteinMaxwell equations, and those of the spatial tracefree parts, respectively (see e.g. [26, 28, 29]). As for coordinate conditions, maximal slicing $K=0$ and the spatially transverse condition (Dirac gauge) $\stackrel{\circ}{D}_{b} \tilde{\gamma}^{a b}=0$ are imposed, where $K$ is the trace of the extrinsic curvature $K_{a b}:=-\frac{1}{2 \alpha}\left(\partial_{t}-£_{\beta}\right) \gamma_{a b}$ of a slice $\Sigma_{t} . \stackrel{\circ}{D}_{a}$ is the covariant derivative with respect to the flat metric $f_{a b}$, and $£_{\beta}$ is the Lie derivative along the shift $\beta^{a}$ defined on $\Sigma_{t}$. As discussed in [26, 28, 29], all metric components have Coulomb type fall off if the condition $\partial_{t} \tilde{\gamma}^{a b}=O\left(r^{-3}\right)$ is imposed. For the present case, the stationarity condition $\partial_{t} \tilde{\gamma}^{a b}=0$ is imposed which is the same condition used in our previous computations for BNS data 27]. Finally, we solve for the Cartesian components of the spatial vector and tensor equations for $\beta_{a}$ and $\tilde{\gamma}_{a b}$, which are written as 3 -dimensional Poisson equations with nonlinear sources.

An analogous formulation is applied for solving Maxwell's equations. The electromagnetic 1-form $A_{\alpha}$ is decomposed in $3+1$ variables $\Phi_{\Sigma}=-A_{\alpha} n^{\alpha}$, and $A_{a}=\gamma_{a}^{\alpha} A_{\alpha}$, and the projections of Maxwell's equations onto $\Sigma_{t}$ as well as along the normal $n^{\alpha}$ to $\Sigma_{t}$ are written in terms of $3+1$ variables $\left\{\Phi_{\Sigma}, A_{a}\right\}$. Imposing the stationarity condition $£_{t} A_{\alpha}=03$ and the Coulomb gauge condition $\stackrel{\circ}{D}^{a} A_{a}=0$, Maxwell's equations reduce to Poisson equations for $\left\{\Phi_{\Sigma}, A_{a}\right\}$ with nonlinear sources.

As mentioned above, gauge conditions for the metric and the electromagnetic fields consist of the maximal slicing $K=0$, the generalized Dirac gauge condition $\stackrel{\circ}{D}_{b} \tilde{\gamma}^{a b}=0$, and the Coulomb gauge condition $\stackrel{\circ}{D}^{a} A_{a}=0$. The condition $K=0$ can be imposed explicitly by replacing $K_{a b}$ with its tracefree part $A_{a b}=$ $K_{a b}-\frac{1}{3} \gamma_{a b} K$ in the above equations. To impose the other two gauge conditions, we introduce the gauge potentials $\xi^{a}$ for the Dirac gauge and $\xi$ for the Coulomb gauge, with which the field variables are transformed as $\tilde{\gamma}^{a b \prime}=\tilde{\gamma}^{a b}-\stackrel{\circ}{D}^{a} \xi^{b}-\stackrel{\circ}{D}^{b} \xi^{a}+\frac{2}{3} f^{a b} \stackrel{\circ}{D}_{c} \xi^{c}$ and $A_{a}^{\prime}=A_{a}-\stackrel{\circ}{D}_{a} \xi$, and let $\tilde{\gamma}^{a b \prime}$ and $A_{a}^{\prime}$ satisfy the gauge conditions [27]. For the case of Coulomb gauge, a substitution of $A_{a}^{\prime}$ to the gauge condition yields an elliptic equation for the gauge potential $\xi, \stackrel{\circ}{D}_{a} \stackrel{\circ}{D}^{a} \xi=\stackrel{\circ}{D}^{a} A_{a}$. This equation and an analogous one for $\xi^{a}$ are solved simultaneously with the field equations at each cycle of iteration, and then the fields $\tilde{\gamma}^{a b}$ and $A_{a}$ are modified according to the above gauge transformations 4

The ideal MHD system of equations includes the MHDEuler equation, the rest mass conservation equation, and

\footnotetext{
3 The Lie derivative $£_{t}$ along the vector $t^{\alpha}$ is defined on $\mathcal{M}$.

${ }^{4}$ In the formulation [28, 29], the Dirac gauge condition is imposed on Einstein's equation explicitly to isolate two independent variables to be solved from dynamic equations.
} 
the perfect conductivity condition. The numerical integration of these partial differential equations, from which time derivative terms are eliminated in order to obtain equilibrium solutions, is technically challenging. Under the assumptions of stationarity and axisymmetry, however, one can find a set of integrability conditions and algebraic relations that the fluid variables must satisfy. Such a formulation for the system of relativistic ideal MHD equations is found in [10, 11, 13], and a fully covariant geometric formulation is derived in our previous work [15]. We rewrite the formulation in [10, 11, 13] as suited to our numerical method. We introduce the basis, $\left\{t^{\alpha}, \phi^{\alpha}, e_{A}^{\alpha}\right\}$, associated with the coordinates $t, \phi$ and two other spatial coordinates $x^{A}$, where $t$ and $\phi$ are chosen along the symmetry vectors $t^{\alpha}$ and $\phi^{\alpha}$ and normalized as $t^{\alpha} \nabla_{\alpha} t=1, \phi^{\alpha} \nabla_{\alpha} \phi=1$, and $e_{A}^{\alpha} \nabla_{\alpha} x^{B}=\delta_{A}{ }^{B}$, where $\delta_{A}^{B}$ is the Kronecker delta. For example, the electric current density $j^{\alpha}$ expanded on this basis is denoted by $j^{\alpha}=j^{t} t^{\alpha}+j^{\phi} \phi^{\alpha}+j^{A} e_{A}^{\alpha}$, and the 4 -velocity by $u^{\alpha}=u^{t} t^{\alpha}+u^{\phi} \phi^{\alpha}+u^{A} e_{A}^{\alpha}$. We also introduce the projection tensor $\sigma_{a}{ }^{b}=\gamma_{a}{ }^{b}-\phi^{b} \nabla_{a} \phi$ : any spatial tensor is projected onto a $\phi=$ constant surface via $\sigma_{A}{ }^{a}$.

For a relativistic magnetized (and charged) perfect fluid with infinite conductivity, the flow always becomes isentropic. From the isentropic flow condition, the rest mass conservation equation, and the projection of the perfect conductivity conditions along the symmetry vectors $t^{\alpha}$ and $\phi^{\alpha}$, we derive conditions for the entropy $S$, the weighted stream function of meridional flow $\sqrt{-g} \Psi$, and the $t$ and $\phi$ components of the electromagnetic potential 1-form $A_{\alpha}$, to be functions of a master potential $\Upsilon, S=S(\Upsilon), \sqrt{-g} \Psi=[\sqrt{-g} \Psi](\Upsilon)$ 占 $A_{t}=A_{t}(\Upsilon)$ and $A_{\phi}=A_{\phi}(\Upsilon)$, which will be referred to as integrability conditions 6 The remaining meridional components of the perfect conductivity condition and MHD-Euler equations (together with the normalization of the 4-velocity) yield a set of algebraic relations that we call first integrals. As a consequence of the two symmetries, any exact 2 form does not have $t \phi$-component.

A common strategy for computing stationary axisymmetric equilibria of the matter and electromagnetic fields under the above integrability conditions is to derive the master equation, called transfield equation, for the master potential $\Upsilon$ by eliminating redundant variables from the first integrals and the Maxwell equations. The transfield equation may be further simplified to a well-known Grad-Shafranov equation by imposing $\Upsilon=A_{\phi}$ and $\sqrt{-g} \Psi=$ constant. Either relation is an elliptic equation, defined on a $\phi=$ constant plane, to be solved for the potential $\Upsilon$ or $A_{\phi}$ (see e.g. [10, 11, 13, 15]).

\footnotetext{
${ }^{5}$ See Eq. [6] for a definition of the stream function for the meridional velocity fields $u^{A}$.

${ }^{6}$ The integrability itself does not constrain the relation between each quantity of $S, \sqrt{-g} \Psi, A_{t}, A_{\phi}$, and the master potential $\Upsilon$, although a solution may not always exist for a certain relations that chosen arbitrarily, or a certain range of parameters involved in the relations.
}

In our formulation, we do not solve such a master equation, but instead solve the first integrals and Maxwell's equations simultaneously. For the sources of Maxwell's equations, we rewrite the current density $j^{\alpha}$ using the first integrals derived from the MHD-Euler equation, hereafter assuming $\Upsilon=A_{\phi}$ for simplicity,

$$
\begin{gathered}
j^{A} \sqrt{-g}=\left([\sqrt{-g} \Psi]^{\prime \prime} h u_{\phi}+\left[\sqrt{-g} \Lambda_{\phi}\right]^{\prime}\right) \delta^{A B} B_{B} \\
-[\sqrt{-g} \Psi]^{\prime} \delta^{A B} \omega_{B}, \\
j^{\phi} \sqrt{-g}+A_{t}^{\prime} j^{t} \sqrt{-g}=\left([\sqrt{-g} \Psi]^{\prime \prime} h u_{\phi}+\left[\sqrt{-g} \Lambda_{\phi}\right]^{\prime}\right) B_{\phi} \\
-[\sqrt{-g} \Psi]^{\prime} \omega_{\phi}-\left(A_{t}^{\prime \prime} h u_{\phi}+\Lambda^{\prime}\right) \rho u^{t} \sqrt{-g},
\end{gathered}
$$

where $B_{B}$ and $\omega_{B}$ are defined by $F_{A \phi}=\partial_{A} A_{\phi}=$ $-\epsilon_{A}^{B} B_{B}$ and $d(h \underline{\mathrm{u}})_{A \phi}=\partial_{A}\left(h u_{\phi}\right)=\epsilon_{A}{ }^{B} \omega_{B}$, respectively, $\epsilon^{A B}$ is an anti-symmetric tensor whose signature is defined as $\epsilon^{12}=-1$, and $B_{\phi}$ is defined by $\epsilon_{A B} B_{\phi}=F_{A B}$. Here, $F_{A \phi}$, and $F_{A B}$ are the components of the Faraday tensor $F_{\alpha \beta}=(d A)_{\alpha \beta}, u^{\alpha}$ is the 4-velocity, $\rho$ the rest mass density and $h=(\epsilon+p) / \rho$ the relativistic enthalpy, where $\epsilon$ is the energy density and $p$ is the pressure. In the above current, $\Lambda$ and $\sqrt{-g} \Lambda_{\phi}$ are arbitrary functions of $\Upsilon\left(=A_{\phi}\right.$ for present case $)$ appearing in the first integrals of the $t$ and $\phi$ components of the MHD-Euler equation. As shown in Eq. (2), the $t$ and $\phi$ components of the 4current are not independent. This is a consequence of one of the integrability conditions that relate the $A_{t}$ and $A_{\phi}$ components to each other, $A_{t}=A_{t}\left(A_{\phi}\right)$. We use the $t$-component of Maxwell's equations to determine $j^{t}$, which is written $4 \pi \alpha j^{t}=D_{a} F^{a}=\psi^{-6} \stackrel{\circ}{D}_{a}\left(\psi^{6} F^{a}\right)$. The components $F^{a}=\gamma_{\alpha}^{a} F^{\alpha \beta} n_{\beta}$ of the Faraday tensor $F_{\alpha \beta}$ involve the prescribed function $A_{t}\left(A_{\phi}\right)$. The calculated quantity $j^{t}$ is then substituted into Eq. (2) to obtain $j^{\phi}$ for the source, in order to integrate the spatial components of Maxwell's equations. It should be noted that, in the perfect MHD case, the current $j^{\alpha}$ is not an independent variable; we introduce $j^{\alpha}$ as an auxiliary variable to derive a formulation suitable for our numerical integration scheme.

Assuming a one-parameter equation of state $p=p(\rho)$, we choose the relativistic enthalpy $h$ as the only independent thermodynamic variable. The matter variables $\left\{h, u^{\alpha}\right\}$ are solved respectively from a first integral of the MHD-Euler equation, the normalization of 4-velocity $u_{\alpha} u^{\alpha}=-1$, the integrability condition of the perfect conductivity condition, and the rest mass conservation equation, as

$$
\begin{aligned}
& h=\Lambda\left(u_{t}-A_{t}^{\prime} u_{\phi}\right)^{-1} \\
& u^{t}=\left[-g_{\alpha \beta}\left(t^{\alpha}+v^{\alpha}\right)\left(t^{\beta}+v^{\beta}\right)\right]^{-1} \\
& u^{\phi}=\frac{[\sqrt{-g} \Psi]^{\prime} B_{\phi}}{A_{\phi}^{\prime} \rho \sqrt{-g}}-\frac{A_{t}^{\prime}}{A_{\phi}^{\prime}} u^{t} \\
& u^{A}=\frac{1}{\rho \sqrt{-g}} \epsilon^{A B} \partial_{B}(\sqrt{-g} \Psi) .
\end{aligned}
$$

At each level of the self-consistent iteration, these equations are used to update hydrodynamic variables $\left\{h, u^{\alpha}\right\}$ by substituting values from the previous iteration level to the right hand sides. 
Finally, to close our formulation for the present calculations, we set arbitrary functions following Newtonian studies on magnetized rotating stars 30]. The functions $A_{t}\left(A_{\phi}\right), \Lambda\left(A_{\phi}\right),\left[\sqrt{-g} \Lambda_{\phi}\right]\left(A_{\phi}\right)$, and $[\sqrt{-g} \Psi]\left(A_{\phi}\right)$ are chosen as follows:

$$
\begin{aligned}
& A_{t}\left(A_{\phi}\right)=-\Omega_{c} A_{\phi} \\
& \Lambda\left(A_{\phi}\right)=-\Lambda_{c} A_{\phi}-\mathcal{E} \\
& {\left[\sqrt{-g} \Lambda_{\phi}\right]\left(A_{\phi}\right)=\frac{a_{\Lambda}}{k+1}\left(A_{\phi}-A_{\phi}^{\max }\right)^{k+1} \Theta\left(A_{\phi}-A_{\phi}^{\max }\right),(9)} \\
& {[\sqrt{-g} \Psi]\left(A_{\phi}\right)=\frac{a_{\Psi}}{q+1}\left(A_{\phi}-A_{\phi}^{\max }\right)^{q+1} \Theta\left(A_{\phi}-A_{\phi}^{\max }\right),(10)}
\end{aligned}
$$

where $\Omega_{c}, \Lambda_{c}, \mathcal{E} a_{\Lambda}, k, a_{\Psi}$, and $q$ are constants, $A_{\phi}^{\max }$ is the maximum value of $A_{\phi}$ at the stellar surface, and $\Theta(x)$ is the Heaviside function. In the above choice of functions, the field $A_{\phi}$ is always positive. The constants $\Lambda_{c}$, and $a_{\Lambda}$ are parameters given by hand and control the magnetic field strength. The constant $a_{\Psi}$ and the exponent $q$ control the meridional flow, where the value of $a_{\Psi}$ is set to a small value so that the flow does not affect the equilibrium of the star, and $q$ is set to one. As discussed in [30], the choice of Eq. (7) corresponds to rigid rotation in the limit of $a_{\Psi} \rightarrow 0$ and $B_{\phi} \rightarrow 0$. Any other choice of the term as a function of $A_{\phi}$ yields differential rotation in the same limit. In [30], it is found that the solutions have comparable strength in the poloidal and toroidal magnetic field components when the index is about $k=0.1$. $\Omega_{c}$ and $\mathcal{E}$ are calculated from conditions imposed on the solution, which fix the values of central density and axis ratio of the deformed star.

The above set of equations (elliptic equations for the gravitational and electromagnetic fields and algebraic relations for the hydrodynamic variables) are numerically solved by self-consistent iteration [31]. In this iteration scheme, the elliptic equations are recast into integral equations using the Green function for the flat Laplacian.

The code is developed as part of the COCAL (Compact Object CALculator) code introduced in 32. In this code, the Einstein-Maxwell and Maxwell's equations for the fields are solved without explicitly imposing axisymmetry. This allows future extensions of the code to compute non-axisymmetric structures such as magnetized NS with tilted poloidal magnetic fields. In the numerical solutions presented below, the star is covered using $161 \times 193 \times 49$ equidistant grid points in $(r, \theta, \phi) \in[0, R(\theta, \phi)] \times[0, \pi] \times$ $[0,2 \pi]$. The space $\Sigma$ is covered by a computational domain $(r, \theta, \phi) \in\left[0,10^{6} R_{0}\right] \times[0, \pi] \times[0,2 \pi]$ with 385 radial non-equidistant grid points and the same equidistant $\theta$ and $\phi$ grids, where $R(\theta, \phi)$ represents the stellar surface, and $R_{0}=R(\pi / 2, \phi)$ the equatorial radius.

Further details of the formulation and numerical method will be described separately in a forthcoming paper.

Numerical solutions: We use the relativistic virial theorem for perfect fluid Einstein-Maxwell spacetimes derived in 33 to study the accuracy of solutions, as well as to quantify the amount of electromagnetic energy. The relativistic virial theorem is an integral that identically vanishes,

$$
\begin{aligned}
& \int_{\Sigma}\left(T_{a}{ }^{a}-\frac{1}{8 \pi} G_{a}{ }^{a}\right) d V \\
& \quad=2 T+3 \Pi+\mathcal{M}+W+M_{\mathrm{ADM}}-M_{\mathrm{K}}=0
\end{aligned}
$$

where $G_{a b}$ and $T_{a b}$ are the projections of the Einstein and stress-energy tensors to a hypersurface $\Sigma$. For stationary spacetimes, an equality of the ADM mass and the Komar mass $M_{\mathrm{ADM}}=M_{\mathrm{K}}$ has been established in 34] (also see e.g. 26]). The quantities $T, \Pi, \mathcal{M}$, and $W$ correspond to the kinetic, internal, electromagnetic, and gravitational energies in the Newtonian limit and are defined by

$$
\begin{aligned}
& T=\frac{1}{2} \int_{\Sigma}(\epsilon+p) u_{a} u^{a} d V, \quad \Pi=\int_{\Sigma} p d V \\
& \mathcal{M}=\frac{1}{16 \pi} \int_{\Sigma}\left(2 F_{a} F^{a}+F_{a b} F^{a b}\right) d V \\
& W=\frac{1}{4 \pi} \int_{\Sigma}\left[\psi^{-4}\left(2 \tilde{D}^{a} \ln \psi \tilde{D}_{a} \ln \psi-\tilde{D}^{a} \ln \alpha \tilde{D}_{a} \ln \alpha\right)\right. \\
& \left.+\frac{3}{4}\left(A_{a b} A^{a b}-\frac{2}{3} K^{2}\right)+\frac{1}{\alpha} K \beta^{a} \tilde{D}_{a} \ln \alpha+\frac{1}{4}{ }^{3} \tilde{R} \psi^{-4}\right] d V,(1
\end{aligned}
$$

where $F_{a b}=\gamma_{a}^{\alpha} \gamma_{b}{ }^{\beta} F_{\alpha \beta}$ is the projection of the Faraday tensor onto $\Sigma$. $A_{a b}$ and $K$ are the tracefree and trace parts of the extrinsic curvature $K_{a b}$, and the tilded quantities $\tilde{D}$ and ${ }^{3} \tilde{R}$ are associated with the conformal 3metric $\tilde{\gamma}_{a b}$. The magnetic energy term $\mathcal{M}$ contains contributions from the poloidal and toroidal magnetic fields, for which we respectively define

$$
\mathcal{M}_{\mathrm{pol}}=\frac{1}{16 \pi} \int_{\Sigma} F_{A B} F^{A B} d V, \quad \mathcal{M}_{\mathrm{tor}}=\frac{1}{8 \pi} \int_{\Sigma} F_{A \phi} F^{A \phi} d V .
$$

Quantities including those defined above for selected compact star solutions with mixed poloidal and toroidal magnetic fields are listed in Table [1] and corresponding contours in $x z(y=0)$-plane are presented in Fig. 1. In these models, we choose a polytropic equation of state $p=K \rho^{\Gamma}$ with an adiabatic constant $\Gamma=2$, and set the parameters in the integrability conditions to $\left(\Lambda_{c}, a_{\Lambda}\right)=$ $(0.8,0.01)$ and $(0.72,0.03)$. In spite of the smallness of the ratio $T /|W|$ compared to $M /|W|$, the stars are significantly deformed; the magnetic fields affect the hydrostatic equilibrium configuration. In these models, the maximum values of the poloidal $B$ fields are above $10^{18} \mathrm{G}$ in cgs Gauss units, $\left(B_{\text {pol }}^{\max }, B_{\text {tor }}^{\max }\right)=\left(1.69 \times 10^{18} \mathrm{G}, 3.11 \times\right.$ $\left.10^{17} \mathrm{G}\right)$ and $\left(1.27 \times 10^{18} \mathrm{G}, 7.56 \times 10^{17} \mathrm{G}\right)$ respectively.

The value of the virial integral $I_{\text {vir }}=|2 T+3 \Pi+\mathcal{M}+W|$ presented in Table@indicates the deviation of a solution from magnetic and hydrostationary equilibrium, which may be caused either by the numerical error, the time dependence of the solution, or both. Since stationarity is imposed on the set of equations in our formulation, the value should converge to zero as the resolution increased. Due to limited computational resources, we haven't performed convergence tests for these magnetized models 7 Nonetheless, the fact that the value $I_{\text {vir }} /|W|$ is

\footnotetext{
7 We have performed convergence tests of the virial integral for relativistic rotating stars turning off the magnetic field and re-
} 

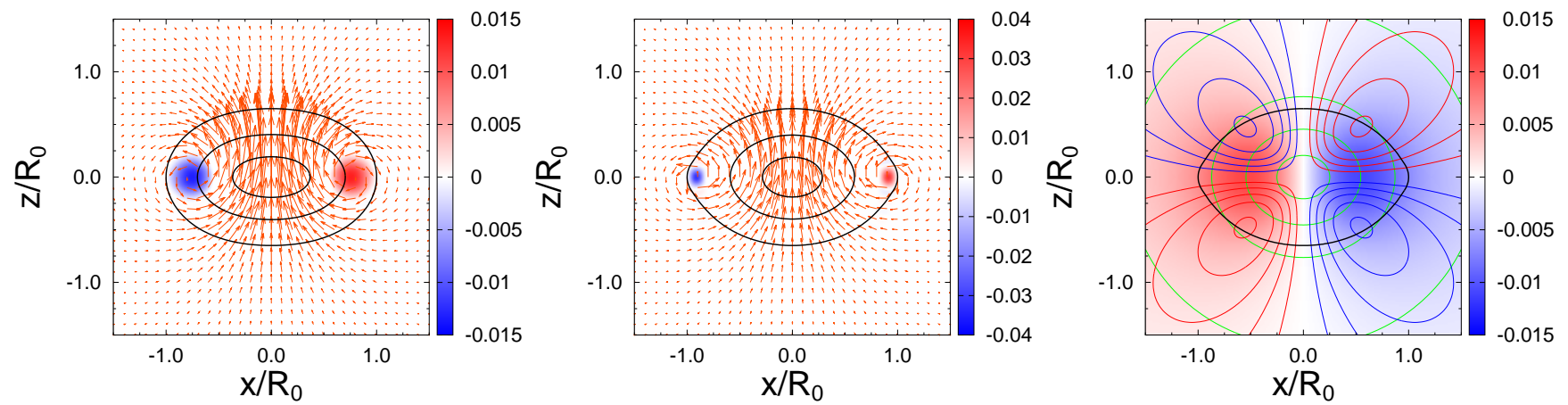

FIG. 1. Left and Center: Contours of $p / \rho$ (black), density map for $B_{\phi}$ (red and blue), and vector plots for $B_{A}$ are shown for models I and II, respectively. Black contours are drawn at the stellar surface and at $p / \rho=0.05$ and 0.1 . Right: Contours for $\psi$ (green), density map for $\tilde{\beta}_{y}$ (red and blue), and contours for $\tilde{\gamma}_{x z}$ (blue and red) are shown for model II. Green contours are drawn in increments of 0.05 , starting from 1.2. Blue (red) contours correspond to negative (positive) values, drawn in increments of 0.002. The thick black oval corresponds to the stellar surface. The maximum values of the magnetic field components for the solutions of the left and center panels are $\left(B_{\text {pol }}^{\max }, B_{\text {tor }}^{\max }\right)=\left(7.19 \times 10^{-2}, 1.32 \times 10^{-2}\right)$ and $\left(5.40 \times 10^{-2}, 3.21 \times 10^{-2}\right)$ respectively.

\begin{tabular}{|c|c|c|c|c|c|c|c|c|c|c|c|c|}
\hline Model & $R_{0}$ & $R_{p} / R_{0}$ & $\overline{p / \rho}$ & $M_{0}$ & $M_{\mathrm{ADM}}$ & $J / M_{\mathrm{ADM}}$ & $T /|W|$ & $\Pi /|W|$ & $\mathcal{M} /|W|$ & $\mathcal{M}_{\mathrm{pol}} /|W|$ & $\mathcal{M}_{\text {tor }} /|W|$ & $I_{\text {vir }} /|W|$ \\
\hline I & 11.8 & 0.662 & 0.122 & 1.788 & 1.551 & 0.236 & $6.17 \mathrm{E}-03$ & $3.05 \mathrm{E}-01$ & $4.69 \mathrm{E}-02$ & $4.51 \mathrm{E}-02$ & $8.69 \mathrm{E}-04$ & $2.48 \mathrm{E}-02$ \\
\hline II & 12.6 & 0.667 & 0.122 & 1.725 & 1.511 & 0.191 & $4.04 \mathrm{E}-03$ & $3.04 \mathrm{E}-01$ & $5.17 \mathrm{E}-02$ & $4.90 \mathrm{E}-02$ & $2.05 \mathrm{E}-03$ & $2.70 \mathrm{E}-02$ \\
\hline
\end{tabular}

TABLE I. Values of physical quantities of selected solutions. Quantities with dimensions are in $G=c=M_{\odot}=4 \pi \varepsilon_{0}=1$ units (e.g. $\left.1 M_{\odot}=145,790 \mathrm{~cm}\right) . M_{0}$ is the rest mass and $I_{\text {vir }}=|2 T+3 \Pi+\mathcal{M}+W|$ is the virial integral.

smaller than $\mathcal{M} /|W|$ suggests that magnetohydrostationary equilibrium is attained in the presented solutions.

The magnetic field becomes stronger as the parameter $\Lambda_{c}$ increases. The magnitude of the toroidal component $B_{\phi}$ is controlled by $a_{\Lambda}$, but its dependence on $a_{\Lambda}$ is not monotonic. Starting from a small value of $a_{\Lambda}, B_{\phi}$ increases as the value $a_{\Lambda}$ increases, and $B_{\phi}$ concentrates near the equatorial surface.

Discussion: The configuration and magnitude of the magnetic fields in the above equilibrium solutions largely depend on the choice of arbitrarily specifiable functions and associated parameters. With our present choice of the functions (7)-(10) and the chosen range of parameters, the energy of toroidal magnetic fields does not exceed that of poloidal fields. Such was also the case in previous Newtonian studies [30], to which we have referred in choosing the functions (7)-(10). As pointed out in simulations [16], the stable magnetized configurations may consist of nearly equal magnitude of poloidal and toroidal magnetic fields. Some recent studies have succeeded in constructing such models in the slow rotation and weak magnetic field limit [13, 38] or in the Newto-

ducing the number of grid points. We have confirmed 2nd order convergnece for the solutions calculated with the waveless formulation, as well as with the Isenverg-Wilson-Mathews (spatially conformally flat) formulation [28, 35]. In the result by Kiuchi and Yoshida [4] for NS with purely toroidal magnetic field, in which a finite difference scheme is used, a typical value of $I_{\mathrm{vir}} /|W|$ is about an order of magnitude smaller than our result. nian limit [39]. It will certainly be possible to find solutions with the toroidal energy nearly equal to, or stronger than, the poloidal energy in our relativistic calculations by surveying a variety of the arbitrary functions and their large parameter space. We will perform such systematic calculations elsewhere.

In our formulation for the Einstein-Maxwell and Maxwell's equations, we only set the time derivatives to zero and recast them in elliptic equations. This does not guarantee a solution to be stationary in general. Imposition of symmetries with respect to vectors $t^{\alpha}$ and $\phi^{\alpha}$ on the set of ideal MHD equations guarantees that the solutions are exact stationary and axisymmetric ones. For more interesting nonaxisymmetric quasiequilibrium data, such as a magnetized NS whose magnetic axis is tilted with respect to the rotation axis or a magnetized BNS, a part of the present code for computing the metric and electromagnetic field can be used as it is 8 As in our previous work for BNS [27], such a computation reproduces initial data in which the radiation content is discarded. With a rather minor extension of the code, we could also compute solutions associated with stationary radiations.

The solutions presented in this paper are useful as initial data sets for numerical simulations [17], as well as unperturbed states for linear stability analysis (see

8 It is necessary to develop another formulation, such as [36, 37], and routines to solve it for computing magnetized quasiequilibriums. 
e.g. [18]). The search for stable and realistic magnetized neutron star models is the next step of this work.

\section{ACKNOWLEDGEMENTS}

We gratefully acknowledge support under JSPS Grantin-Aid for Scientific Research(C) 23540314 and 25400262,
Grant-in-Aid for Scientific Research on Innovative Areas 24103006, DFG grant SFB/Transregio 7 "Gravitational Wave Astronomy" and STFC grant PP/E001025/1.
[1] R. C. Duncan and C. Thompson, Astrophys. J. 392, L9 (1992).

[2] B. D. Metzger, D. Giannios, T. A. Thompson, N. Bucciantini and E. Quataert, Mon. Not. Roy. Astron. Soc. 413, 2031 (2011)

[3] M. Bocquet, S. Bonazzola, E. Gourgoulhon and J. Novak, Astron. Astrophys. 301, 757 (1995)

[4] K. Kiuchi and S. Yoshida, Phys. Rev. D 78, 044045 (2008)

[5] J. Frieben and L. Rezzolla, Mon. Not. Roy. Astron. Soc. 427, 3406 (2012)

[6] B. Carter, in Black holes - Les Houches 1972, edited by C. DeWitt \& B.S. DeWitt, Gordon and Breach, New York (1973), p. 57;

[7] A. Oron, Phys. Rev. D 66, 023006 (2002);

[8] J. L. Friedman and N. Stergioulas, Rotating Relativistic Stars, Cambridge University Press, Cambridge, UK, 2013.

[9] N. Straumann, General Relativity, Springer Science+Business Media Dordrecht, 2013.

[10] J. D. Bekenstein and E. Oron, Phys. Rev. D, 18, 1809 (1978);

[11] K. Ioka and M. Sasaki, Phys. Rev. D 67, 124026 (2003); K. Ioka and M. Sasaki, Astrophys. J. 600, 296 (2004)

[12] R. Ciolfi, V. Ferrari, L. Gualtieri and J. A. Pons, Mon. Not. Roy. Astron. Soc. 397, 913 (2009); R. Ciolfi, V. Ferrari and L. Gualtieri, Mon. Not. Roy. Astron. Soc. 406, $2540(2010)$

[13] S. Yoshida, K. Kiuchi and M. Shibata, Phys. Rev. D 86, 044012 (2012)

[14] A. G. Pili, N. Bucciantini and L. Del Zanna, Mon. Not. Roy. Astron. Soc., 439, 3541 (2014)

[15] E. Gourgoulhon, C. Markakis, K. Uryu and Y. Eriguchi, Phys. Rev. D 83, 104007 (2011)

[16] J. Braithwaite and H. C. Spruit, Nature 431, 819 (2004); J. Braithwaite and Å. Nordlund, A\&A 450, 1077 (2006); J. Braithwaite, A\&A 453, 687 (2006); J. Braithwaite, A\&A 469, 275 (2007) J. Braithwaite, MNRAS 397763 (2009); V. Duez, J. Braithwaite, and S. Mathis, ApJ 724 L34 (2010).

[17] M. Shibata and Y. -i. Sekiguchi, Phys. Rev. D 72, 044014 (2005); M. D. Duez, Y. T. Liu, S. L. Shapiro, M. Shibata and B. C. Stephens, Phys. Rev. D 73 104015, (2006); B. Giacomazzo and L. Rezzolla, Class. Quant. Grav. 24, S235 (2007); M. Shibata, Y. Suwa, K. Kiuchi, and K. Ioka, ApJL 734, L36 (2011); S. L. Liebling, L. Lehner, D. Neilsen and C. Palenzuela, Phys. Rev. D 81, 124023 (2010); T. Kuroda and H. Umeda, Astrophys. J. Suppl. 191, 439 (2010); K. Kiuchi, S. Yoshida and M. Shibata, Astron. Astrophys. 532, A30 (2011); K. Kiuchi, K. Kyutoku and M. Shibata, Phys. Rev. D 86, 064008 (2012);
Z. B. Etienne, V. Paschalidis, Y. T. Liu and S. L. Shapiro, Phys. Rev. D 85, 024013 (2012)

[18] S. Yoshida, Phys. Rev. D 86, 104055 (2012)

[19] A. Papapetrou, Ann. Inst. Henri Poincare Phys. Theor. IV , 83 (1966).

[20] W. Kundt and M. Trumper, Z. Phys. 192, 419 (1966).

[21] S. Chandrasekhar, The Mathematical Theory of Black Holes, New York : Oxford University Press, 1992.

[22] R. M. Wald, General Relativity, University of Chicago Press, Chicago, 1984.

[23] See Supplemental Material at [URL] for a brief review on stationary and axisymmetric spacetimes for rotating and magnetized compact stars.

[24] R. Birkl, N. Stergioulas and E. Muller, Phys. Rev. D 84, 023003 (2011)

[25] E. Gourgoulhon, and S. Bonazzola, Phys. Rev. D, 48, 2635 (1993)

[26] M. Shibata, K. Uryu and J. L. Friedman, Phys. Rev. D 70, 044044 (2004) [Erratum-ibid. D 70, 129901 (2004)]

[27] K. Uryu, F. Limousin, J. L. Friedman, E. Gourgoulhon and M. Shibata, Phys. Rev. Lett. 97, 171101 (2006); K. Uryu, F. Limousin, J. L. Friedman, E. Gourgoulhon and M. Shibata, Phys. Rev. D 80, 124004 (2009).

[28] E. Gourgoulhon : 3+1 Formalism in General Relativity; Bases of Numerical Relativity, Springer (Berlin) (2012)

[29] S. Bonazzola, E. Gourgoulhon, P. Grandclement and J. Novak, Phys. Rev. D 70, 104007 (2004) gr-qc/0307082.

[30] S. Yoshida, and Y. Eriguchi, ApJS, 164, 156 (2006); S. Yoshida, S. Yoshida, and Y. Eriguchi, Astrophys. J., 651, 462 (2006)

[31] J. P. Ostriker, and J. W.-K. Mark, Astrophys. J.151, 1075 (1968); I. Hachisu, ApJS 62, 461 (1986); ibid. 61, 479 (1986); H. Komatsu, Y. Eriguchi, and I. Hachisu, Mon. Not. Roy. Astron. Soc. 237, 355 (1989)

[32] X. Huang, C. Markakis, N. Sugiyama and K. Uryu, Phys. Rev. D 78, 124023 (2008) K. Uryu and A. Tsokaros, Phys. Rev. D 85, 064014 (2012) K. Uryu, A. Tsokaros and P. Grandclement, Phys. Rev. D 86, 104001 (2012)

[33] E. Gourgoulhon, and S. Bonazzola, Classical and Quantum Gravity, 11, 443 (1994)

[34] R. Beig, Physics Letters A, 69, 153 (1978)

[35] See Supplemental Material at [URL] for a convergence test of COCAL code on the virial integral for relativistic rotating stars.

[36] J. D. Bekenstein and G. Betschart, Phys. Rev. D 74, 083009 (2006)

[37] K. Uryu, E. Gourgoulhon and C. Markakis, Phys. Rev. D 82, 104054 (2010)

[38] R. Ciolfi and L. Rezzolla, Mon. Not. Roy. Astron. Soc., 435, L43 (2013) 
[39] K. Fujisawa and Y. Eriguchi, Mon. Not. Roy. Astron. Soc., 432, 1245 (2013) 